\title{
Especies del género Malassezia en piel sana de niños y adolescentes con VIH/SIDA
}

\section{(Malassezia species on healthy skin of children and teens with HIV / AIDS)}

\author{
Brenda López U*, Ana Castillo G, Naylu Montilla A, Sandra Robertiz Ch, \\ Priscila Fernández C, María Del monte V, Evelyn González-Moran. \\ Laboratorio de Micología, Escuela de Bioanálisis, Facultad de Medicina. \\ Universidad del Zulia-Venezuela. \\ *Autor para correspondencia: bplu88@gmail.com
}

RECIBIDO:06 de Septiembre de 2017 APROBADO:21 de Noviembre de 2017

DOI: 10.22370/bolmicol.2017.32.2.973

LOS AUTORES DECLARAN NO TENER CONFLICTO DE INTERESES

Palabras claves: Colonización; piel; Malassezia; VIH.

Key words: Colonization; Skin; Malassezia; HIV.

\section{RESUMEN}

Antecedentes: Las especies del género Malassezia forman parte de la biota cutánea normal humana y pueden ser aisladas en áreas ricas en glándulas sebáceas. Su colonización es alta durante edad prepuberal y puberal debido al aumento de actividad de dichas glándulas. Objetivo: Determinar la colonización por especies del género Malassezia en piel sana de niños y adolescentes con VIH/SIDA. Metodología: Las muestras fueron tomadas mediante el método de la impronta con cinta adhesiva transparente, en cuero cabelludo, pabellón auricular, pecho, espalda, muslo, antebrazo, palma de la mano e inoculadas en el medio con base de goma Spondia dulcis. La identificación de las especies se realizó siguiendo las claves descritas por Guého et al. Se realizó el test de difusión en tween propuesto por Guillot et al, la prueba de la catalasa, y la utilización de triptófano como fuente única de nitrógeno. Resultados: De un total de 80 niños y adolescentes, solo $23(28.75 \%)$ de ellos se les aisló Malassezia. 10 (43.48\%) de sexo masculino y $13(56.52 \%)$ de sexo femenino. Solo se encontró, Malassezia sympodialis con un predominio del $100 \%$. Correspondiendo al mayor porcentaje de positividad al grupo etario de 4-7 años (56.52\%). Las localizaciones anatómicas predominantes fueron: pabellón auricular (25.5\%) seguida de pecho $(21.3 \%)$ y espalda (19.1\%). Conclusiones: En niños con HIV/SIDA se observa un patrón de colonización por M. sympodialis.

\section{ABSTRACT}

Background: Malassezia species are part of the normal human skin biota and can be isolated from different body areas, mainly those rich in sebaceous glands. Colonization is high during prepuberal and puberal for the increased activity of the sebaceous glands. Aims: Determine the colonization by Malassezia species in healthy skin of children 
and teenagers with HIV/AIDS. Methods: Samples were taken using imprint method with transparent adhesive tape, were taken from scalp, ear, chest, back, thigh, forearm and palm; were inoculated in Spondias dulcis medium. The species identification was performed according the instructions described by Guého et al, Tween diffusion test proposed by Guillot et al, catalase test, and the use of tryptophan as only source of nitrogen. Results: From a total of 80 children and teenagers, only 23 $(28.75 \%)$ of them were isolated Malassezia. 10 $(43.48 \%)$ were from males and $13(56.52 \%)$ from females. Found only Malassezia sympodialis with a prevalence of $100 \%$. Corresponding to the higher percentage of positivity the age group 4-7 years $(56.52 \%)$. The predominant anatomical locations were: ear $(25.5 \%)$ followed by chest $(21.3 \%)$ and back (19.1\%) respectively. Conclusions: In children with HIV / AIDS colonization pattern observed is the present of M. sympodialis.

\section{INTRODUCCIÓN}

La colonización fúngica de la piel por levaduras del género Malassezia tiene una amplia distribución en áreas ricas en glándulas sebáceas (pecho, oídos, espalda, cara y cuero cabelludo), ya que estas levaduras son en su mayoría lipofílicas, pues presentan un defecto en la capacidad de sintetizar ácidos grasos saturados[1][2]. M. pachydermatis es la única especie de Malassezia no lipodependiente, siendo considerada como una levadura zoofílica, ya que ha sido aislada en piel y el conducto auditivo externo de animales silvestres y domésticos[3]. Los lípidos presentes en la piel humana son capaces de satisfacer los requerimientos de estos organismos, observándose durante la pre pubertad y pubertad un incremento de la colonización, debido al aumento de la actividad de las glándulas sebáceas [1][4].

Bajo la influencia de ciertos factores, las levaduras de Malassezia pueden volverse patógenas y ocasionar diversas enfermedades. Los factores predisponentes endógenos incluyen la piel grasa, hiperhidrosis, herencia, tratamiento con corticosteroides, mal nutrición e inmunodeficiencias. Mientras que los factores exógenos más importantes son temperatura y la humedad relativa elevada. En países tropicales las condiciones climáticas favorecen la colonización por Malassezia con respecto a los países europeos y asiáticos donde se ha observado una baja frecuencia[5]. Malassezia es agente etiológico de pitiriasis versicolor y foliculitis por Malassezia, esta se asocia a dermatitis seborreica y exacerba otros procesos dermatológicos como la dermatitis atópica, psoriasis, acné vulgaris, dacriocistitis, blefaritis seborreica, pustulosis neonatal, papilomatosis reticular y confluente, entre otros[6] [7]. Las especies de Malassezia han sido relacionadas con procesos sistémicos, habiéndose aislado de peritonitis, tejido pulmonar y, especialmente, en hemocultivo de sujetos con catéteres venosos centrales y sometidos a alimentación parenteral[8][9].

La piel es el órgano que con mayor frecuencia se altera en la infección por el VIH, y desde la introducción del tratamiento antirretroviral de gran actividad (TARGA) han existido diversas modificaciones de las manifestaciones cutáneas de estos pacientes[10]. A pesar de que aún se desconoce la etiología de la enfermedad, Malassezia ha sido asociada al desarrollo de dermatitis seborreica (SD) en pacientes con VIH, encontrando relación entre el mayor número de levaduras presentes en las lesiones y el bajo recuento de linfocitos $\mathrm{T}$ CD4[11][13]. El objetivo de este estudio fue caracterizar la colonización de piel sana por especies del género Malassezia en niños y adolescentes con VIH/SIDA.

\section{MATERIALES Y MÉTODOS}

Se realizó un estudio a 80 niños y adolescentes con VIH/SIDA con edades comprendidas entre 1 a 15 años, los cuales asistían periódica- 
mente a la consulta de la fundación INNOCENS del Hospital Universitario de Maracaibo, Estado Zulia. Los pacientes bajo estudio tenían un diagnóstico confirmado a través de pruebas de laboratorio, según algoritmo de la organización mundial de la salud (OMS)[14] y ausencia de infecciones dermatológicas como pitiriasis versicolor, dermatitis seborreica, foliculitis, dermatitis atópica, psoriasis, acné vulgaris entre otras. Aquellos pacientes que habían recibido tratamientos con antibióticos y/o antimicóticos tres meses previos a la toma de las muestras fueron excluidos.

Las muestras de piel sana se tomaron de 7 localizaciones anatómicas; 6 regiones ricas en glándulas sebáceas como son: cuero cabelludo (CC), pabellón auricular (PA), pecho (PE), espalda (E), antebrazo (AB) y muslo (M). Adicionalmente se muestreo en palma de la mano (PM), debido a que $M$. pachydermatis no es lipofílica, reportada presente en esta localización asociada a dueños de animales domésticos[15][16]. Se empleó el método de la impronta con cinta adhesiva transparente. De cada una de las localizaciones en estudio se obtuvo dos muestras, con una se realizó un examen directo con Azul de metileno al 0,25\% observándose al microscopio con objetivo de 40X. Con la otra cinta se procedió a realizar el cultivo, colocando la cinta con la impronta en las placas de Petri con medio Spondia dulcis con cloranfenicol y se incubaron a $32^{\circ} \mathrm{C}$ por 14 días. Las placas que no presentaron crecimiento característico de Malassezia en el tiempo estipulado se reportaron como negativas. Las colonias compatibles con Malassezia spp se subcultivaron en medio Spondia dulcis sin cloranfenicol para su identificación[17][18].

Las especies de Malassezia se tipificaron en base a los criterios descritos por Guého et al[19]; el test de difusión en tween propuesto por Guillot et al[20], prueba de catalasa, y la utilización de triptófano como única fuente de nitrógeno. Un amplio número de aislados clínicos obtenidos de estudios realizados anteriormente en nuestro laboratorio fueron empleados como referencia[21]-[23].

Los resultados obtenidos en la presente investigación fueron analizados a través del programa estadístico SPSS vol 17, en donde se aplicó un análisis de tablas de frecuencias absolutas. En la identificación de las diferencias estadísticas de las variables estudiadas se realizaron tablas de contingencia y se efectuaron las Pruebas de Chi cuadrado y el análisis de corrección de Spearman y de Pearson. Se tomó el $99.99 \%$ como índice de confiabilidad estadístico $(\mathrm{p}<0,0001)$.

\section{RESULTADOS}

En el estudio realizado a una población de 80 niños y adolescentes de la fundación INNOCENS del Hospital Universitario de Maracaibo, 23 pacientes $(28.75 \%)$ fueron positivos para Malassezia. Es importante resaltar, que en cada paciente se tomó muestra de 7 localizaciones anatómicas, donde se logró aislar por paciente en una o más localizaciones, obteniendo un total de 47 aislados. E1 100\% de los aislados correspondieron a Malassezia sympodialis.

Al evaluar la presencia de M. sympodialis según los grupos etarios, se observó un predominio en niños con edades comprendidas entre 4-7 años 13 (56.52\%), seguido por el grupo de edades entre los 1-3 años 5 (21.74\%), mientras que en los grupos de 8 -11 años 3 (13.04\%) y de 12-15 años $2(8.70 \%)$, no presentaron prevalencia significativa. En la distribución de $M$. sympodialis según el sexo de los niños y adolescentes evaluados no hubo diferencias estadísticamente significativas, sexo masculino $10(43.48 \%)$ y sexo femenino 13 $(56.52 \%)$.

Se determinó que de los 47 aislados obtenidos en las diferentes localizaciones anatómicas $M$. sympodialis fue aislada en mayor predominio en 
pabellón auricular $12(25.5 \%)$, seguida por pecho $10(21.3 \%)$, espalda $9(19.1 \%)$, cuero cabelludo 7 $(14.9 \%)$ y muslo, antebrazo y palma de la mano 3 $(6.4 \%)$ respectivamente.

\section{DISCUSIÓN}

El género Malassezia forma parte de la biota normal en la piel humana, y actualmente ha adquirido gran importancia dentro de los patógenos emergentes, debido a las recurrentes infecciones sistémicas causadas por estos hongos[1].

En este trabajo se estudiaron 80 pacientes con edades comprendidas de 1-15 años, diagnosticados con VIH/SIDA, en donde solo 23 (28.75\%) fueron positivos para Malassezia. Si bien, el agar Dixon es conocido ampliamente por permitir el aislamiento de Malassezia, el medio de cultivo empleado ha demostrado tener buen rendimiento, sin embargo, el uso de esta alternativa podría estar vinculado con el bajo aislamiento[17]. El método utilizado para la toma de muestra es el recomendado para muestreo en piel sana, asimismo existen otros métodos como son raspado de escamas con bisturí y el uso de hisopo estéril, los cuales son empleados principalmente para toma de muestra de lesiones, no obstante, la presencia de lesión en nuestros pacientes se considera un criterio de exclusión. En estudios comparativos no se reporta una diferencia significativa sobre el diferente uso de técnicas para la toma de muestra de Malassezia, por lo cual no se considera que el uso del método de la cinta adhesiva transparente este asociado con el bajo número de aislamientos[24]-[26].

Los resultados obtenidos coincidieron con estudios realizados por Gupta et al [4] en donde el $36.3 \%$ de los individuos entre 0-14 años fueron positivos para Malassezia, así mismo el estudio realizado por González et al (2011)[21] en niños en edad preescolar (2 a 7 años), que presentaron un $36.3 \%$ de aislamiento. Sin embargo nuestros resultaron difieren de los obtenidos por Bergbrant y Broberg[27], en donde los pacientes con edades comprendidas entre los 0-14 años presentaron un $87 \%$ de aislamientos, así como también difieren de los resultados del estudio de González et al (2009) [22] con niños desnutridos de 1 a 6 años de edad presentaron una prevalencia de $65.9 \%$.

El género Malassezia se considera levaduras oportunistas emergentes de gran importancia; especialmente presente en pacientes inmunosuprimidos. En pacientes con VIH/SIDA se ha observado el desarrollo de diversas enfermedades dermatológicas, no obstante, SD severas han sido encontradas en mayor proporción, especialmente en aquellos resistentes al tratamiento y en estadios avanzados de la enfermedad, caracterizada por eritema, placas redondeadas con comezón y presencia de escamas en cuero cabelludo, cara e ingle[24] [28] [30].

Al evaluar la prevalencia de Malassezia, el 100\% de los 47 aislados obtenidos, correspondieron a Malassezia sympodialis. Estos datos coinciden con diferentes reportes[31]-[33] que señalan a $M$. sympodialis como la especie más común en piel sana, debido a que es nutricionalmente menos selectiva que otras. Sin embargo, este dato no coincide con los aislados identificados en otras investigaciones[21]-[23] donde la mayoría de los aislados correspondieron a Malassezia furfur. Nuestros datos también se diferencian de otros estudios realizados en pacientes adultos con VIH, en donde la especie con mayor número de aislamientos es $\mathrm{Ma}$ lassezia pachydermatis [3][34].

En relación a la edad se ha observado que la colonización de la piel sana por especies de $M a$ lassezia comienza durante las primeras semanas de vida, alcanzando niveles del $30 \%$ al cabo de un mes[35]. En niños menores de 10 años la colonización es baja, pero durante la edad prepuberal y puberal, debido al aumento de la actividad de 
las glándulas sebáceas, la colonización es mayor (80-90 \%)[4]. Sin embargo, los datos obtenidos en nuestro estudio difieren ya que la mayoría de los aislamientos se obtuvieron en niños menores de 7 años $(78.26 \%)$. A pesar de que en la edad puberal normalmente se aísla un alto porcentaje de Malassezia debido a la alta actividad de las glándulas sebáceas, los resultados obtenidos no se correlacionan ya que solo se consiguieron 2 aislamientos $(8.70 \%)$ en el grupo de $12-15$ años, es importante referir que este grupo etario estaba conformado por un bajo número de pacientes. No se determinaron diferencias estadísticas significativas de colonización por Malassezia entre el sexo masculino y el femenino. El rol del sexo y su relación con la colonización por Malassezia aún no está bien definido, nuestros resultados tienen similitud con otros estudios donde no se encuentra esta asociación[36]-[38]. La correlación entre las especies de Malassezia, edad y sexo de los sujetos ha sido observada con una baja recuperación en infantes a discrepancia de lo que ocurre en la pubertad[23], pero sin diferencias entre mujeres y hombres.

Los resultados obtenidos en la presente investigación señalan que la colonización de piel sana de niños con VIH/SIDA está dada por $M$. sympodialis, con un predominio de los aislamientos en pabellón auricular (25.5\%) y pecho (21.3\%). Se ha señalado que, en individuos sanos, las especies de Malassezia varían de acuerdo con la región corporal estudiada. Sosa et al describen mayor aislamiento de diversas especies de Malassezia incluyendo M. sympodialis en cuero cabelludo[39]. Mientras que otros estudios realizados en nuestro laboratorio[21][23][40] se observa una mayor frecuencia de aislados de Malassezia en espalda, seguido de pabellón auricular.

Es de gran importancia continuar las investigaciones sobre estas levaduras, en piel sana y enferma en otros grupos etarios de pacientes con VIH/SIDA, que permitan establecer que factores influyen en su prevalencia.

\section{AGRADECIMIENTOS}

Fundación INNOCENS y al Prof. Jorge Guiñez de la Facultad Experimental de Ciencias de la Universidad del Zulia.

\section{REFERENCIAS}

1. G. Giusiano. Malassezia: Estado del conocimiento y perspectivas en su estudio. Rev. Argent. Microbiol. 2006; 38: 41-48.

2. K. Harada, M. Saito, T. Sugita, R. Tsuboi. Malassezia species and their associated skin diseases. J. Dermatol. 2015; 42(3): 250-257.

3. R. Cruz, P. Vieille, L. Carvajal. Malassezia pachydermatis en lesiones de piel en pacientes de la Quinta región de Chile. Rev. argentina dermatología. 2014; 95(4): 1-5.

4. A. K. Gupta \& Y. Kohli. Prevalence of $M a-$ lassezia species on various body sites in clinically healthy subjects representing different age groups. Med. Mycol. 2004; 42: 35-42.
5. J. Faergemann. Atopic dermatitis and fungi. Clin. Microbiol. Rev.2002; 15(4): 545-563.

6. L. Angiolella, S. Carradori, C. Maccallini, G. Giusiano, C. T. Supuran. Targeting Malassezia species for Novel Synthetic and Natural Antidandruff Agents. Curr. Med. Chem. 2017; 24(22): 2392-2412.

7. A. Velegraki, C. Cafarchia, G. Gaitanis, R. Iatta, T. Boekhout. Malassezia Infections in Humans and Animals: Pathophysiology, Detection, and Treatment. Plos Pathog. 2015; 11(1): e1004523.

8. C. Aguirre, C. Euliarte, J. Finquelievich, M. de los Á. Sosa, G. Giusiano. Fungemia and in- 
terstitial lung compromise caused by Malassezia sympodialis in a pediatric patient. Rev. Iberoam. Micol. 2015; 32(2):118-121.

9. A. Tragiannidis, G. Bisping, G. Koehler, A. H. Groll. Minireview: Malassezia infections in immunocompromised patients. Mycoses. 2010; 53(3): 187-195.

10. D. Lastrada. Manifestaciones cutáneas de la infección por VIH-SIDA. Medicas UIS. 2006; 19 : $191-200$.

11. R. C. Schechtman, G. Midgley, R. J. Hay. HIV disease and Malassezia yeasts: a quantitative study of patients presenting with seborrhoeic dermatitis. Br. J. Dermatol. 2006; 133(5): 694-698.

12. Y. Amado et al. Seborrheic dermatitis: predisposing factors and ITS2 secondary structure for Malassezia phylogenic analysis. Med. Mycol. 2013; 51(8): 868-875.

13. World Health Organization. "Interim who clinical staging of HIV/AIDS and HIV/AIDS case definitions for surveillance african region" Geneva 2005.

14. M. Unemo, R. Ballard, C. Ison, D. Lewis, F. Ndowa, R. Peeling. Diagnóstico de laboratorio de las infecciones de transmisión sexual, incluida la infección por el virus de la inmunodeficiencia humana. Organización Panamericana de la Salud 2014.

15. D. O. Morris, K. O'Shea, F. S. Shofer, S. Rankin. Malassezia pachydermatis carriage in dog owners. Emerg. Infect. Dis. 2005; 11(1): 83-88.

16. M. Zuiani, J. Amadeo. Búsqueda de Malassezia spp. en personal hospitalario en contacto con pacientes oncohematologicos. Acta Bioquím Clín Latinoam. 2006; 40(2): 223-227.

17. L. Mesa et al. Comparison of the growth of
Malassezia furfur and Malassezia slooffiae on Spondias dulcis gum exudate and Dixon media. Kasmera. 2008; 36(1): 45-52.

18. L. M. Mesa C, O. Urdaneta, S. Rodríguez de Valero, V. Fernández, G. León de Pinto, R. Villalobos. Comportamiento de Malassezia furfur en medios de cultivo con base en los exudados gomosos de Spondias dulcis y Spondias mombin. Producción de lipasa extracelular. Kasmera. 2011; 39(1): 18-25.

19. E. Guého, G. Midgley, J. Guillot. The genus Malassezia with description of four new species. Antonie Van Leeuwenhoek. 1996; 69: 337-355.

20. J. Guillot, E. Gueho, G. Midgley, G. Chevrier, B. Dupont. Identification of Malassezia species. A practical approach. J. Mycol. Med. 1996; 6:103-110.

21. E. Gonzalez, M. Delmonte, S. Robertiz, P. Fernandez, L. Mesa, S. Rodriguez. Colonización de especies de Malassezia en piel sana de niños en edad preescolar. Kasmera. 2011;38(2): 107-113.

22. E. González-Morán et al. Aislamiento e identificación de especies del género Malassezia en piel sana de niños desnutridos y eutróficos de multihogares de cuidado diario. Invest. Clin. 2009; 50(2): 145-152.

24. P. Fernandez, E. Gonzalez, M. Delmonte, S. Robertiz. Caracterización de especies de $\mathrm{Ma}$ lassezia en piel sana de estudiantes de secundaria.

Kasmera. 2014; 42(1): 66-73.

25. J. Roth. Common cutaneous complications of HIV disease. PRN Noteb. 1997; 2(4): 7-10.

26. H. Hussein, M. Ahmad, M. Hamed, M. Hassan. Comparative study for the reliability of cellophane tape and standard $\mathrm{KOH}$ mount in diagnosis of pityriasis versicolor. Gulf J. Dermatology Venereol. 2010; 17(2): 29-34. 
27. A.Omodo-Eluk, K. Baker, H. Fuller. Comparison of Two Sampling Techniques for the Detection of Malassezia pachydermatis on the Skin of Dogs with Chronic Dermatitis. Vet. J. 2003; 165(2): 119-124.

28. I. Bergbrant \& A. Broberg. Pityrosporum ovale culture from the forehead of healthy children. Acta Derm Venereol. 1994; 74(4): 260-261.

29. S. Rodriguez et al. Tratamiento antirretroviral de gran actividad en niños VIH positivos . Evolución de la enfermedad relacionada con parámetros clínicos, inmunológicos y virológicos al comienzo del tratamiento. Arch Argent Pediatr. 2009; 107(3): 212-220.

30. L. Jerez Puebla, C. Fernández, M. Illnait, M. Perurena, I. Rodríguez, G. Martínez. Diagnóstico micológico en pacientes infectados por el Virus de la Inmunodeficiencia Humana. Arch. Venez. Farmacol. y Ter. 2010; 29(2): 55-58.

31. K. Altman, E. Vanness, and R. P. Westergaard. Cutaneous Manifestations of Human Immunodeficiency Virus: a Clinical Update. Curr Infect Dis Rep. 2015; 17(3): 464.

32. V. Crespo, A. Ojeda, Á.Vera, A. Crespo, F. Sanchez. Aislamiento e identificacion de Malassezia spp en pitiriasis versicolor, dermatitis seborreica y piel sana. Rev. Iberoam. Micol. 1999; 16: $16-21$.

33. A. Gupta, Y. Kohli, R. C. Summerbell, J. Faergemann. Quantitative culture of Malassezia species from different body sites of individuals with or without dermatoses. Med Mycol. 2001; 39(3): 243-251.

34. M. H. Sandström Falk et al. The prevalence of Malassezia yeasts in patients with atopic dermatitis, seborrhoeic dermatitis and healthy controls. Acta Derm. Venereol. 2005; 85(1): 17-23.

35. R. Cruz, P. Rodríguez, R. Novoa. Micosis mucocutáneas en pacientes con VIH-Sida de la ciudad de Valparaíso, Chile. Rev. argentina dermatología. 2013; 94(4): 2-5.

36. T. Juncosa Morros et al. Colonización cutánea neonatal por Malassezia spp. An Esp Pediatr. 2002; 57(5): 452-456.

37. Y. W. Lee et al. Distribution of Malassezia species on the scalp in korean seborrheic dermatitis patients. Ann. Dermatol. 2011; 23(2): 156-161.

38. A. Prohić, T. Jovović Sadiković, S. Kuskunović-Vlahovljak, R. Baljić. Distribution of Malassezia Species in Patients with Different Dermatological Disorders and Healthy Individuals. Acta Dermatovenerol. Croat. 2016; 24(4): 274-281.

39. A. Prohic, D. Simic, T. J. Sadikovic, M. Krupalija-Fazlic. Distribution of Malassezia species on healthy human skin in Bosnia and Herzegovina: correlation with body part, age and gender. Iran. J. Microbiol. 2014; 6(4): 253-262.

40. M. Sosa, F. Rojas, M. Mangiaterra, G. Giusiano. Prevalencia de especies de Malassezia asociadas a lesiones de dermatitis seborreica en pacientes de Argentina. Rev. Iberoam. Micol. 2013; 30(4): 239-242.

41. S. Rodriguez, L. Mesa, E. Gonzalez, M. Delmonte, S. Robertiz, A. Valero. Caracterización fenotípica de especies de Malassezia en piel sana de población estudiantil universitaria. Invest. Clin. 2005; 46(4): 329-335. 